\title{
Increased Within-Network Functional Connectivity May Predict NEDA Status in Fingolimod-Treated MS Patients
}

\author{
Claudia Piervincenzi ${ }^{1}$, Nikolaos Petsas ${ }^{2 *}$, Laura De Giglio ${ }^{3}$, Maurizio Carmellini ${ }^{1}$, \\ Costanza Gianni ${ }^{1}$, Silvia Tommasin ${ }^{1}$, Carlo Pozzilli ${ }^{4}$ and Patrizia Pantano ${ }^{1,2}$
}

' Department of Human Neurosciences, Sapienza University of Rome, Rome, Italy, ${ }^{2}$ Department of Radiology, IRCCS NEUROMED, Pozzilli, Italy, ${ }^{3}$ Neurology Unit, San Filippo Neri Hospital, Rome, Italy, ${ }^{4}$ Multiple Sclerosis Center, S. Andrea Hospital, Department of Human Neurosciences, Sapienza University of Rome, Rome, Italy

Only a few studies have evaluated the brain functional changes associated with disease-modifying therapies (DMTs) in multiple sclerosis (MS), though none used a composite measure of clinical and MRI outcomes to evaluate DMT-related brain functional connectivity (FC) measures predictive of short-term outcome. Therefore, we investigated the following: (1) baseline FC differences between patients who showed evidence of disease activity after a specific DMT and those who did not; (2) DMT-related effects on FC, and; (3) possible relationships between DMT-related FC changes and changes in performance. We used a previously analyzed dataset of 30 relapsing MS patients who underwent fingolimod treatment for 6 months and applied the "no evidence of disease activity" (NEDA-3) status as a clinical response indicator of treatment efficacy. Resting-state $\mathrm{FMRI}$ data were analyzed to obtain within- and between-network FC measures. After therapy, 14 patients achieved NEDA-3 status (hereinafter NEDA), while 16 did not (EDA). The two groups significantly differed at baseline, with the NEDA group having higher within-network FC in the anterior and posterior default mode, auditory, orbitofrontal, and right frontoparietal networks than the EDA. After therapy, NEDA showed significantly reduced within-network FC in the posterior default mode and left frontoparietal networks and increased between-network FC in the posterior default mode/orbitofrontal networks; they also showed PASAT improvement, which was correlated with greater within-network FC decrease in the posterior default mode network and with greater between-network FC increase. No significant longitudinal FC changes were found in the EDA. Taken together, these findings suggest that NEDA status after fingolimod is related to higher within-network FC at baseline and to a consistent functional reorganization after therapy.

Keywords: multiple sclerosis, resting-state functional MRI, functional connectivity, fingolimod, no evidence of disease activity 


\section{INTRODUCTION}

Multiple sclerosis (MS) is an immune-system-mediated disease characterized by inflammation, demyelination, and degeneration of the central nervous system (CNS), leading to a deterioration in life quality and expectancy (1). Disease-modifying therapies (DMTs) have multiplied in the last 2 decades, with new categories of more targeted and effective drugs (2). Clinical trials have proven the efficacy of these treatments for relapsing MS in terms of clinical relapse rate, disability progression, and MRI-assessed lesion accumulation (3).

Multiple advanced MRI techniques have been used to investigate the mechanisms of action and the efficacy of DMTs in MS. Progress has also been made in the investigation of DMT effects on the brain in terms of tissue loss, microstructural abnormalities, metabolic changes, and functional plasticity [for a review, see (4)]. Over the years, functional MRI (fMRI) has provided important insights into MS-damage-related plasticity and functional reorganization, although only a few studies have evaluated brain functional changes associated with DMTs in MS. Of these, 2 studies used motor-task-evoked fMRI to investigate therapy-related (IFN- $\beta$ 1a) functional activity changes in the sensorimotor network $(5,6)$. Even less is known about resting-state functional connectivity (FC) changes after DMT. With respect to task-based fMRI, resting-state fMRI is more advantageous in clinical studies because it is easily applicable, allows the simultaneous investigation of different brain networks, and avoids the confounding effects of disability in performing a given behavioral task $(7,8)$.

In our recent study, we investigated $\mathrm{FC}$ changes of the primary motor cortex in relapsing MS after 6 months of fingolimod treatment (Gilenya ${ }^{\circledR}$; Novartis) (9). Using a seedbased approach, we found a significant decrease in FC between this region and posterior cortical areas, which correlated with a significant cognitive improvement as measured by the Paced Auditory Serial Addition Task (PASAT). However, in that study, we directly explored changes only in the sensorimotor network through a seed-based analysis of the primary motor cortex. The other resting-state networks (RSNs), including those directly cognition related, were not investigated as that would have been beyond the scope of the study. Moreover, treatment efficacy was measured in terms of conventional MRI parameters (gadolinium-positive lesions and new T2 lesions), although the attempt to use them as grouping criterion did not lead to significant results. Thus, our previous results have suggested the need to investigate large-scale network connectivity to better understand DMT-related brain functional rearrangement and FC measures that predict treatment outcome. Therefore, the present study aims to (1) identify baseline FC differences between patients who showed evidence of disease activity after a specific DMT and those who did not; (2) investigate the longitudinal effect of DMT on FC among responders and non-responders, and (3) identify possible relationships between FC changes and DMT-related changes in performance.

For this purpose, we used the dataset from our previous study (9) on relapsing MS patients who underwent fingolimod treatment for 6 months. After that period of time, we used the "no evidence of disease activity" (NEDA-3) status characterization as a clinical response indicator of treatment efficacy $(2,10)$. We hypothesized that FC before and during treatment reflects a brain status that would determine susceptibility to DMT action on the basis of neuroinflammatory and/or neuroplasticity mechanisms. In this framework, NEDA status achievement would be reflected in DMT-induced functional reorganization at the level of large-scale brain networks joined to the antiinflammatory effect of DMT. Furthermore, we also hypothesized that the clinical relevance of FC changes would be reflected in their correlation with concomitant changes in behavioral measures. To confirm this hypothesis, we expected the following outcomes: (1) prediction of NEDA status based on baseline FC and (2) a correlation between FC longitudinal changes after 6 months of DMT and significantly changed behavioral measures.

\section{MATERIALS AND METHODS}

\section{Participants}

All patients belonged to a previously published prospective, post-marketing, interventional study [for details, see (9)], in which a consecutive series of 36 patients with a diagnosis of relapsing-remitting MS (11) were recruited at Sant'Andrea Hospital, Sapienza University of Rome, Italy and underwent an MRI scan and clinical testing at Policlinico Umberto I, Sapienza University of Rome, Italy. Thirty-two patients completed the protocol and were included in the previous analyses; this cohort constituted our initial dataset. We analyzed MRI images acquired 1-2 days prior to starting fingolimod [therapy start (Tst)] and after 6 months of fingolimod treatment (T6m). Two patients were excluded from the present study because of a missing postgadolinium $\mathrm{T} 1$ sequence. Of the remaining 30 patients, 4 were treatment naïve, 20 switched from a previous firstline, and 6 switched from a second-line DMT. The switch from first-line DMT was due to inefficacy while that from the second line (Natalizumab) was due to Progressive Multifocal Leukoencephalopathy risk. Patients were then divided into groups according to the NEDA-3 composite outcome measure (10). Patients who achieved NEDA-3 status (hereinafter referred to as NEDA) were defined as those who had: (1) no relapses, (2) no confirmed disability progression at 6 months, and (3) no new/enlarged T2 or gadolinium positive lesions. Patients who did not achieve NEDA were defined as EDA.

At each time point and before being scanned, participants were assessed by a trained physician and the following clinical outcomes were collected: Expanded Disability Status Scale (EDSS) score (12), MS Functional Composite (MSFC) (13) score with its subscores [9-Hole Peg Test (9-HPT); 25-Feet Walk Test (25-FWT); and the Paced Auditory Serial Addition Test (PASAT) 3 and 2 s].

\section{Ethics Statement}

This study was performed in accordance with the ethical code of the ethics committee of Azienda Policlinico Umberto I, Sapienza University of Rome, and the Declaration of Helsinki. After approval from the ethics committee, written informed consent was obtained from all subjects. 


\section{MRI Acquisition}

Images were acquired with a 3-Tesla (3T) scanner (Siemens Magnetom Verio) and a 12-channel head coil designed for parallel imaging (GRAPPA). Participants were advised to avoid consuming psychoactive substances, such as tea or coffee, within $2 \mathrm{~h}$ prior to MRI scans.

The following sequences were acquired:

- Blood oxygen level-dependent (BOLD) single-shot echoplanar imaging $\left(\mathrm{TR}=3,000 \mathrm{~ms}, \mathrm{TE}=30 \mathrm{~ms}\right.$, flip angle $=89^{\circ}$, FOV $=192 \mathrm{~mm}, 64 \times 64$ matrix, 50 contiguous axial slices 3 mm thick, 140 volumes, voxel size $=3 \mathrm{~mm}^{3}$, acquisition time $=7 \mathrm{~min} 11 \mathrm{~s}$ ), with all patients instructed to close their eyes and stay awake during the resting-state fMRI acquisitions;

- High-resolution 3D, T1-weighted (T1-3D) MPRAGE sequence $(\mathrm{TR}=1,900 \mathrm{~ms}$, TE $=2.93 \mathrm{~ms}$, TI $=900 \mathrm{~ms}$, flip angle $=9^{\circ}, \mathrm{FOV}=260 \mathrm{~mm}$, matrix $=256 \times 256,176$ sagittal slices $1 \mathrm{~mm}$ thick, no gap);

- Dual turbo spin-echo, proton density (PD) and T2-weighted images $(\mathrm{TR}=3,320 \mathrm{~ms}$, TE1 $=10 \mathrm{~ms}$, TE2 $=103 \mathrm{~ms}$, FOV $=220 \mathrm{~mm}$, matrix $=384 \times 384,25$ axial slices $4 \mathrm{~mm}$ thick, $30 \%$ gap);

- T1-weighted spin-echo sequence acquisition after administration of a gadolinium-based contrast agent ( $\mathrm{TR}=$ $550 \mathrm{~ms}, \mathrm{TE}=9.8 \mathrm{~ms}, \mathrm{FOV}=240 \mathrm{~mm}$, matrix $=320 \times 320$, 25 axial slices $4 \mathrm{~mm}$ thick, $30 \%$ gap).

\section{MRI Analysis}

\section{Data Quality}

Image quality metrics were extracted from functional and structural MRI data using the default pipeline of MRIQC (14), an open-source analysis program that estimates image quality metrics (IQMs) to provide interoperability, uniform standards, and to assess reliability of a dataset. Supplementary Tables 1, 2 provide lists of all IQMs obtained with MRIQC, showing that our measures are in line with those of other studies that have provided IQMs (e.g., see http://mriqc.org).

\section{Lesion Load and Brain Volume}

Lesion volume was calculated on the PD images using a semiautomated technique with Jim 5.0 software (Xinapse System, Leicester, UK; http://www.xinapse.com). Measures of global brain volume and gray matter (GM) volume at baseline (i.e., at Tst) were also obtained from lesion-filled T1-3D brain images via SIENAX software, a fully automated method for measuring crosssectional brain volume that is freely available as part of FMRIB's Software Library (FSL) (https://fsl.fmrib.ox.ac.uk/fsl/fslwiki).

\section{Functional Connectivity \\ Data Pre-processing}

Functional MRI data were preprocessed using FSL, version 5.0.9. Single-subject preprocessing was performed using FMRI Expert Analysis Tool (FEAT). Pre-statistical processing consisted of motion correction using MCFLIRT (15), brain extraction using BET (16), slice-timing correction, spatial smoothing using a Gaussian kernel of full-width at half-maximum of $5 \mathrm{~mm}$, and high-pass temporal filtering with a cut-off of $100 \mathrm{~s}$. Registration to high-resolution structural and/or standard space images was performed using FLIRT $(15,17)$. EPI volumes were registered to the individual structural scan using the FLIRT_Boundary-Based Registration (BBR) tool (18). Registration from high-resolution structural to standard space was then further refined using FNIRT non-linear registration (19). 4D GM maps were obtained using the feat_gm_prepare tool and were used as voxel-wise nuisance variables in subsequent statistical analyses.

Independent component analysis (ICA) of preprocessed functional data was performed using Multivariate Exploratory Linear Optimized Decomposition into Independent Components (MELODIC) tool (20). For group-wise ICA, a single $4 \mathrm{D}$ dataset was created by temporally concatenating preprocessed functional data containing 140 time points for each subject. The dimensionality of group-ICA was performed using different numbers of components (i.e., 20, 25, 30, 35, 40) (20-22). Finally, a dimensionality of 25 was chosen, as the explained data variance $(45.95 \%)$ was sufficient to obtain good estimates of the signals, and well-known RSNs were identified (21). RSNs of interest were identified via spatial correlation coefficients $(f s l c c$ tool) using RSNs generated by Smith et al. (21) and Yeo et al. (23) as templates, and then verified by expert visual inspection (CPi, NP, PP).

The set of spatial maps from the group-average analysis was used to generate subject-specific versions of the spatial maps and associated time series using a dual regression technique $(24,25)$. For each subject, the group-average set of spatial maps was first regressed (as spatial regressors in a multiple regression) into the subject's $4 \mathrm{D}$ space-time dataset, resulting in a set of subjectspecific time series, one per group-level spatial map. These time series were then regressed (as temporal regressors in a multiple regression) into the same $4 \mathrm{D}$ dataset, resulting in a set of subjectspecific spatial maps, one per group-level spatial map. Individual difference maps between $\mathrm{T} 6 \mathrm{~m}$ and Tst ( $\Delta \mathrm{FC}$ maps) were also obtained for each RSN.

\section{Statistical Analyses}

Statistical analyses of demographic, clinical, radiological, and neuropsychological parameters were performed using SPSS statistics software (version 22.0). To investigate the possible confounding role of the above parameters in the prediction of NEDA status, between-group differences at Tst were tested using Mann-Whitney $U$-test and Chi-square test for continuous and dichotomous variables, respectively $(p<$ 0.05 for null hypothesis rejection). In the subgroup of interest (NEDA), longitudinal changes in the neuropsychological scores were estimated using Wilcoxon signed-rank test ( $p<0.05$ for null hypothesis rejection); for significant changes, individual differences between $\mathrm{T} 6 \mathrm{~m}$ and Tst $(\Delta)$ were obtained and used for voxel-wise correlation with $\triangle \mathrm{FC}$ maps.

\section{Head Motion Analysis}

Head motion (even if small) can bias derived results in groups where there are motion differences (26-28). To examine if there are any differences in head motion among NEDA and EDA patients at baseline, or between sessions for each subgroup, absolute and relative displacement 
TABLE 1 | Demographic and clinical characteristics, radiological features, and scores obtained in the clinical/neuropsychological assessment at fingolimod therapy start (Tst) in the 2 patient subgroups.

\begin{tabular}{|c|c|c|c|}
\hline & NEDA $(N=14)$ & $\operatorname{EDA}(N=16)$ & $p^{*}$ \\
\hline \multicolumn{4}{|l|}{ Demographic/clinical features } \\
\hline Age & $37.4 \pm 6.5$ & $36.1 \pm 8.4$ & ns \\
\hline Female/male $(n)$ & $8 / 6$ & $15 / 1$ & 0.018 \\
\hline Disease duration (year) & $10.4 \pm 5.6$ & $10.0 \pm 8.3$ & ns \\
\hline EDSS score [median (range)] & $1.75[1.5-5.0]$ & $2.0[1.0-6.0]$ & ns \\
\hline Relapses in previous year [n (\%)] & $4(29)$ & $9(56)$ & ns \\
\hline Treatment naïve [n (\%)] & $3(21)$ & $1(6)$ & ns \\
\hline First-line/second-line therapy $(n)$ & $8 / 3$ & $12 / 3$ & ns \\
\hline \multicolumn{4}{|l|}{ Radiological features } \\
\hline Brain volume $\left(\mathrm{cm}^{3}\right)$ & $1,464 \pm 68$ & $1,495 \pm 66$ & ns \\
\hline Gray matter volume $\left(\mathrm{cm}^{3}\right)$ & $735 \pm 48$ & $765 \pm 39$ & ns \\
\hline T2-lesion volume $\left(\mathrm{cm}^{3}\right)$ & $14.239 \pm 12.143$ & $6.653 \pm 4.979$ & $\mathrm{~ns}$ \\
\hline Gadolinium-positive lesion [n (\%)] & $5 / 14(36)$ & 10/16 (62) & ns \\
\hline \multicolumn{4}{|c|}{ Clinical/Neuropsychological scores } \\
\hline 9-HPT dominant hand (s) & $20.4 \pm 4.9$ & $19.1 \pm 3.0$ & ns \\
\hline 9-HPT non-dominant hand (s) & $22.3 \pm 7.4$ & $20.8 \pm 4.0$ & $\mathrm{~ns}$ \\
\hline PASAT 3 (s) & $42.5 \pm 13.7$ & $45.4 \pm 12.8$ & $\mathrm{~ns}$ \\
\hline PASAT 2 (s) & $35.1 \pm 15$ & $35.7 \pm 12.9$ & ns \\
\hline 25-FWT (s) & $5.5 \pm 1.2$ & $6.5 \pm 2.1$ & ns \\
\hline
\end{tabular}

Values are reported as the mean \pm standard deviation or median [min-max].

$n$, number; s, seconds; ns, not statistically significant.

${ }^{*} B$ etween-group differences at Tst were tested using Chi-square test (sex, treatmentnaive dichotomous variables) and Mann-Whitney U-test (all other continuous variables) (p $<0.05$ for null hypothesis rejection).

values were obtained by using the McFLIRT tool in FSL (15) and compared using a two-sample unpaired $t$-test and paired $t$-test, respectively, with $p<0.05$ for null hypothesis rejection.

\section{Within-Network Functional Connectivity}

Subject-specific spatial maps obtained from dual regression analysis were entered into group-level voxel-wise analyses. To investigate within-network FC differences at Tst, we compared the 2 patient subgroups using a two-sample unpaired $t$ test.

To investigate within-network FC changes after 6 months of treatment and compare longitudinal changes between the 2 patient subgroups, a two-sample unpaired $t$-test was performed on $\triangle \mathrm{FC}$ maps of NEDA and EDA patients. Age, sex, and GM maps were entered as nuisance variables in all analyses.

Voxel-wise statistical analyses were performed with permutation-based non-parametric statistics using FSL Randomize permutation-based program with 5,000 permutations (29). Clusters were determined by using thresholdfree cluster enhancement (TFCE) (30) and a family-wise error (FWE)-corrected cluster significance threshold of $p<0.05$. The Randomize tool (5,000 permutations) was also used to examine the statistical correlation between significant within-network FC longitudinal changes and behavioral parameters that changed over time. Correlation analyses were performed inside the mask of significant longitudinal FC changes. Resulting statistical maps were thresholded at $p<0.05$, FWE corrected. Anatomical localization of significant clusters was established according to the Harvard-Oxford Cortical Structural Atlas included in FSL (http://www.fmrib.ox.ac.uk/fsl/data/atlas descriptions. html).

\section{Between-Network Functional Connectivity}

Between-network functional connectivity differences were investigated using FSL Nets toolbox (http://fsl.fmrib.ox.ac. $\mathrm{uk} / \mathrm{fs}$ //fslwiki/FSLNets). After normalization of the extracted time courses (output of the first stage of dual regression) of all RSNs identified in each subject, time courses of artifactual components and components of no interest were regressed out of the individual data. Subject-wise correlation matrices of both full and partial correlation of all remaining RSN time courses were then created. Resulting correlation coefficients (connection or edge strengths) were then Fisher z-transformed and corrected for temporal autocorrelation. Between-subject testing was then conducted across correlation values acquired for pairs of independent components.

Between-network connectivity differences at Tst between the 2 patient subgroups were investigated using non-parametric unpaired testing (FSL Randomize tool, 5,000 permutations; age, sex, and GM as nuisance variables). Between-network connectivity differences between Tst and T6m were investigated using non-parametric paired testing. The statistical significance threshold was set at $p<0.05$, FWE corrected. Finally, the relationship between behavioral changes and between-network FC changes ( $\triangle \mathrm{FC}$, expressed as the difference between $\mathrm{T} 6 \mathrm{~m}$ and Tst of the z-transformed correlation coefficients) was assessed using Spearman's rank correlation in SPSS, with $p<0.05$ for null hypothesis rejection, corrected for multiple comparisons.

\section{RESULTS}

\section{Clinical and Conventional MRI Findings}

Descriptive statistics for demographic, clinical, radiological, and neuropsychological parameters at Tst in the 2 patient subgroups are reported in Table $\mathbf{1}$.

After 6 months of therapy, 14 patients achieved NEDA (46.65\%), while 16 did not (53.35\%). In the EDA subgroup, 9 patients had MRI activity, 4 had both clinical and MRI activity, and 3 had disability worsening.

There were no significant differences at Tst (Table 1) between NEDA and EDA in terms of age, disease duration, EDSS score, number of relapses in the previous year, number of treatmentnaive patients, and number of patients switching from firstor second-line DMTs. Regarding radiological characteristics, no significant differences at Tst were found between the 2 subgroups in terms of brain volume, gray matter volume, lesion volume, and the number of gadolinium-enhancing lesions. No significant differences were found between NEDA and EDA patients in terms of neuropsychological scores. The only significant difference between subgroups was gender distribution, with 6 males in the NEDA subgroup vs. one in the EDA subgroup. 

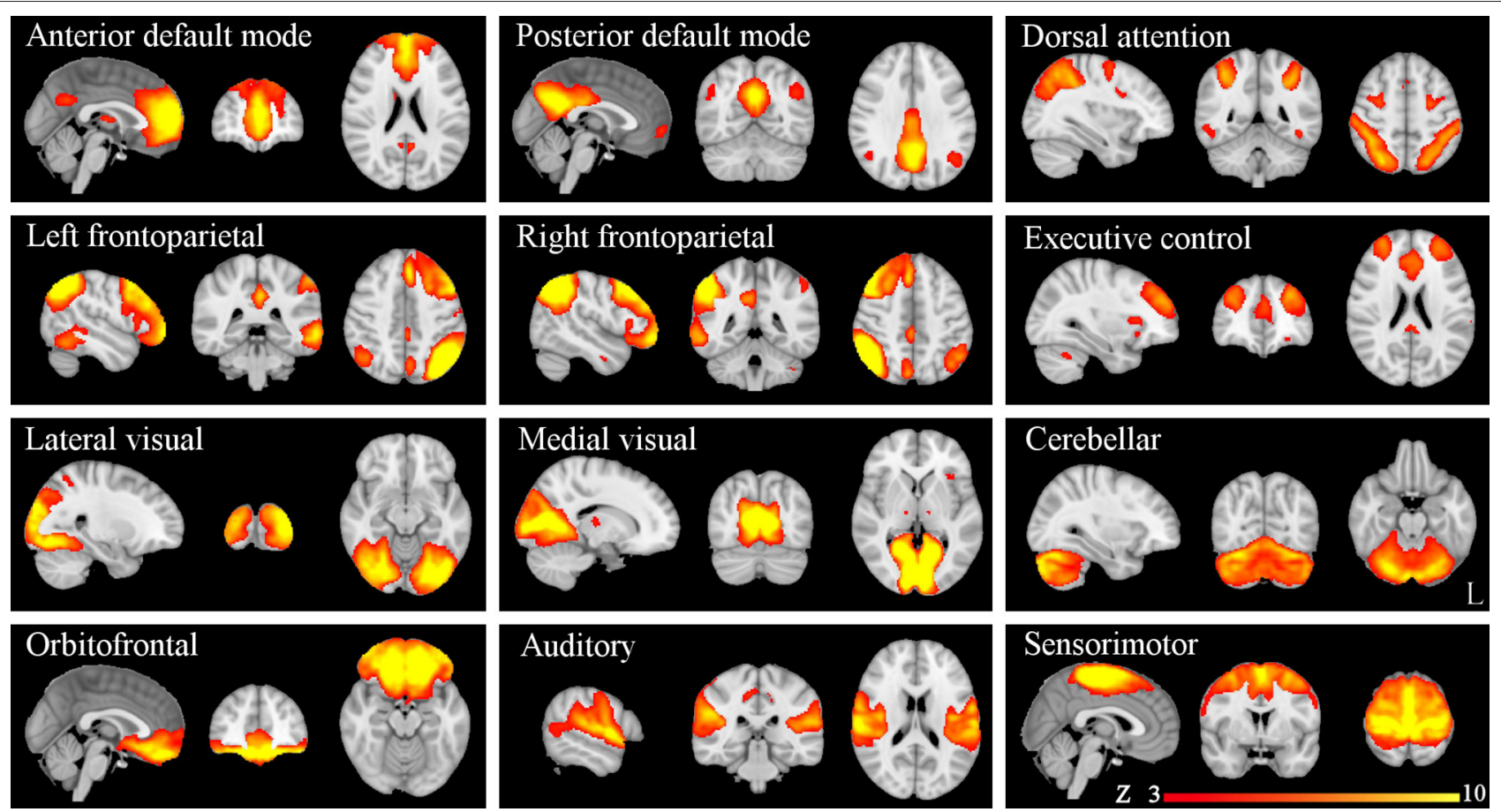

FIGURE 1 | Resting-state networks (RSNs) identified and used for dual regression analysis. This figure shows sagittal, coronal, and axial slices for the RSNs detected, overlaid onto the MNI152 standard brain. RSNs are shown in FSL red-yellow color encoding using a $3<$-score $<10$ threshold window.

In NEDA patients, PASAT scores showed a significant longitudinal improvement after 6 months of therapy (PASAT3: $p=0.033$; PASAT2: $p=0.046$ ), while 9-HPT and 25-FWT scores did not show any significant longitudinal change.

\section{Resting-State Functional Connectivity Head Motion}

At Tst, maximum absolute head motion was $0.53 \mathrm{~mm}$ in the NEDA group and $0.87 \mathrm{~mm}$ in the EDA group. Mean absolute and relative displacement values were $0.24 \pm 0.11$ and $0.05 \pm$ $0.02 \mathrm{~mm}$ in NEDA, $0.25 \pm 0.19$ and $0.04 \pm 0.01 \mathrm{~mm}$ in EDA. At $\mathrm{T} 6 \mathrm{~m}$, maximum absolute head motion was $0.55 \mathrm{~mm}$ in the NEDA group and $0.40 \mathrm{~mm}$ in the EDA group. Mean absolute and relative displacement values were $0.29 \pm 0.16$ and $0.05 \pm$ $0.02 \mathrm{~mm}$ in NEDA and $0.22 \pm 0.08$ and $0.03 \pm 0.01 \mathrm{~mm}$ in EDA. No subject was identified as a motion outlier. There were no significant differences in motion parameters between NEDA and EDA at baseline, nor between sessions in each subgroup.

\section{Within-Network Connectivity}

ICA yielded 25 independent components representing groupaveraged networks of brain regions with BOLD fMRI signals that were temporally correlated. Of these, we identified 12 components that showed the highest spatial correlation coefficients with RSN templates, accounting for $49.9 \%$ of the explained variance and for $22.92 \%$ of the total variance: the anterior and posterior default mode $(r=0.41$ and $r=$ 0.67 , respectively), dorsal attention $(r=0.51)$, left and right frontoparietal ( $r=0.68$ and $r=0.67$, respectively), executive control $(r=0.52)$, lateral visual $(r=0.65)$, medial visual $(r=$ $0.76)$, cerebellar $(r=0.30)$, orbitofrontal $(r=0.40)$, auditory $(r$ $=0.69$ ), and sensorimotor $(r=0.58)$ networks (Figure 1).

At Tst, significant differences in within-network FC were found between patient subgroups. More specifically, NEDA patients showed significantly higher FC with respect to EDA patients in five RSNs, namely the anterior and posterior default mode, orbitofrontal, right frontoparietal, and auditory networks ( $p<0.05$, FWE corrected) (Figure 2; Table 2).

After 6 months of therapy, decreased within-network FC was found in NEDA patients in regions of the posterior default mode and left frontoparietal networks $(p<0.05$, FWE corrected) (Figure 3A; Table 3). No significant FC changes were found in EDA patients after therapy. The comparison between $\triangle F C$ maps of NEDA and EDA patients showed a significant difference in the posterior default mode network ( $p<0.05$, FWE corrected), with NEDA patients showing a significant reduction in FC over time as compared with the EDA group which showed no significant change (Figure 3B; Table 4).

Correlation analyses between significant longitudinal FC changes and behavioral changes in NEDA patients yielded no FWE-corrected results. However, since the number of NEDA patients was low and our previous study on the same cohort already reported a significant correlation between therapyrelated FC changes and cognitive performance (9), statistical results using false-discovery rate (FDR) multiple-comparisons correction $(p<0.05)$ are also reported (Figure 4; Table 5). Voxelwise negative correlations were found in NEDA patients between 

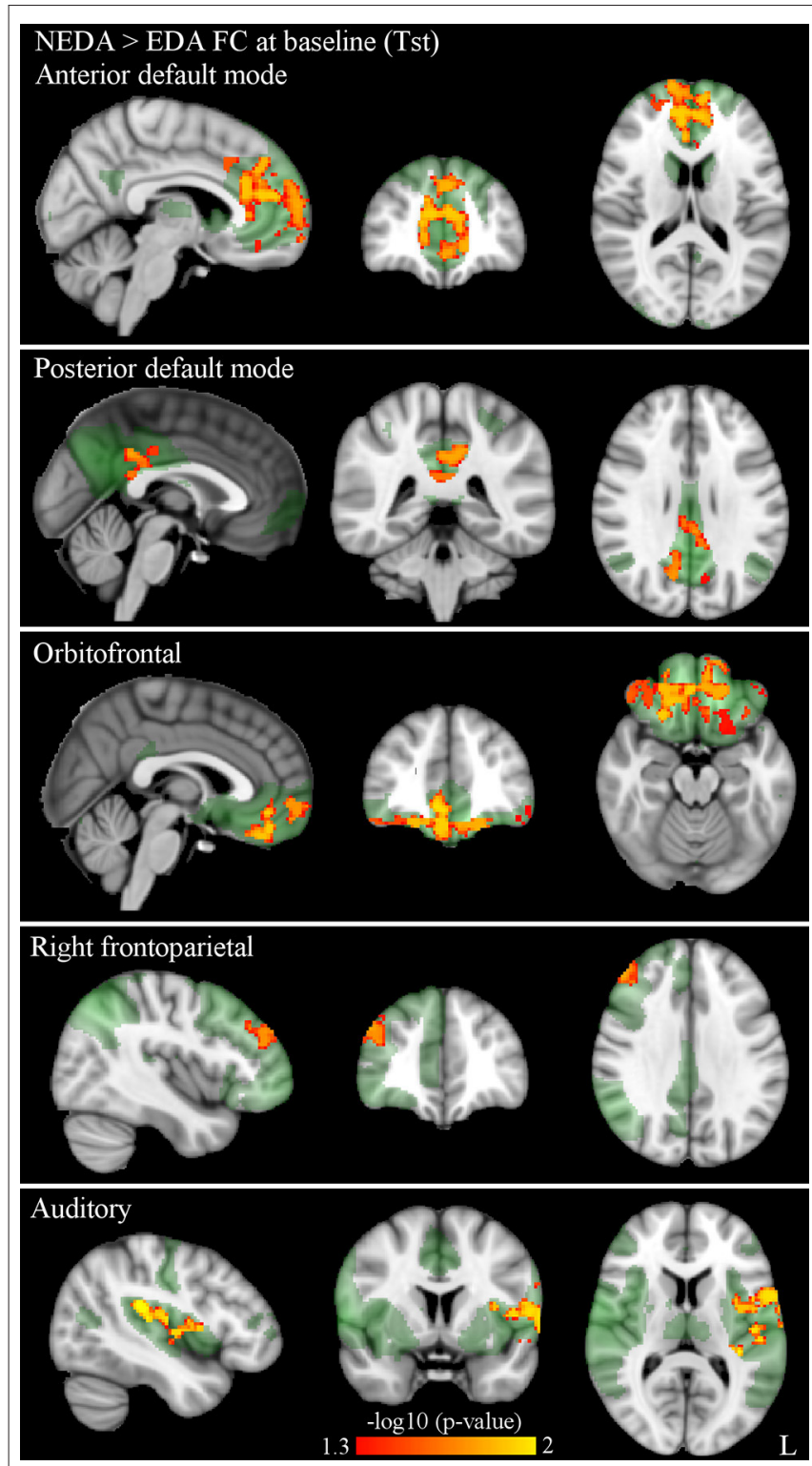

FIGURE 2 | Significantly higher within-network FC in NEDA as compared with EDA patients at baseline in the anterior default, posterior default, orbitofrontal, right frontoparietal, and auditory networks ( $p<0.05$, FWE corrected). Results for each RSN are overlaid onto the corresponding network (green) in the MNI152 standard brain. The red-yellow color bar represents level of significance. The -log10(p)-value represents the negative base-10 logarithm of the $p$ value. A larger value of $-\log 10(p)$ corresponds to a smaller $p$-value.

FC decrease in the posterior default mode network and increase in $\triangle$ PASAT3 and $\triangle$ PASAT2 after therapy. The greater the PASAT increase at 6 months, the greater the FC decrease in the posterior default mode network. No significant correlations were found with the left frontoparietal network.

To address the predictive value of baseline FC for NEDA status, post-hoc logistic regression analyses were performed using SPSS to ascertain the effect of FC on the likelihood of achieving NEDA. In the statistical model, within-network FC was
TABLE 2 | RSNs showing significantly higher within-network FC in NEDA as compared with EDA patients at baseline ( $p<0.05$, FWE corrected).

\begin{tabular}{|c|c|c|c|c|c|}
\hline \multirow[b]{2}{*}{$\begin{array}{l}\text { Cluster size } \\
\text { (voxels) }\end{array}$} & \multirow[b]{2}{*}{$p$} & \multicolumn{3}{|c|}{ MNI coordinates } & \multirow[b]{2}{*}{ Cluster location (local maxima) } \\
\hline & & $x$ & $y$ & $z$ & \\
\hline
\end{tabular}

Anterior default mode network

$\begin{array}{cccccl}2,915 & 0.014 & 8 & 46 & 16 & \text { Paracingulate gyrus } \\ 0.015 & 12 & 66 & 12 & \text { Right frontal pole } \\ 0.015 & -10 & 56 & 10 & \text { Left frontal pole } \\ 0.018 & -2 & 44 & -8 & \text { Paracingulate gyrus } \\ 0.024 & -10 & 42 & 34 & \text { Left superior frontal gyrus }\end{array}$

Posterior default mode network

$\begin{array}{llllll}871 & 0.018 & -4 & -42 & 32 & \text { Cingulate gyrus, posterior division } \\ & 0.019 & 12 & -66 & 26 & \text { Precuneous cortex } \\ 42 & 0.035 & 14 & -34 & 38 & \text { Cingulate gyrus, posterior division } \\ 39 & 0.047 & -14 & -60 & 18 & \text { Precuneous cortex } \\ & 0.047 & -8 & -68 & 24 & \text { Precuneous cortex }\end{array}$

Auditory network

$\begin{array}{cccccl}1,465 & 0.01 & -44 & -30 & 18 & \text { Left parietal operculum cortex } \\ 0.012 & -56 & 6 & 6 & \text { Left precentral gyrus } \\ 0.013 & -42 & -10 & 2 & \text { Left insular cortex }\end{array}$

Orbitofrontal network

$\begin{array}{lccccl}1,868 & 0.011 & 6 & 36 & -10 & \text { Paracingulate gyrus } \\ & 0.014 & 20 & 22 & -24 & \text { Right frontal orbital cortex } \\ & 0.014 & 24 & 52 & -12 & \text { Right frontal pole } \\ & 0.016 & -18 & 42 & -22 & \text { Left frontal pole } \\ & 0.02 & 4 & 56 & -14 & \text { Frontal pole/frontal medial cortex } \\ & 0.046 & -48 & 44 & -12 & \text { Left frontal pole }\end{array}$

Right frontoparietal network

$\begin{array}{lllll}263 & 0.017 & 48 & 38 & 32\end{array}$

Peak MNI coordinates $(\mathrm{mm})$ within clusters were identified using the minimum peak distance between the local maxima of $20 \mathrm{~mm}$. Anatomical localizations of peak $\mathrm{MN}$ coordinates were established according to the Harvard-Oxford Cortical Structural Atlas.

represented by the mean value inside the mask of each RSN, showing a significant connectivity difference between NEDA and EDA patients (auditory, anterior default mode, posterior default mode, orbitofrontal, and right frontoparietal networks). We firstly investigated correlations between within-network FC values of the 5 RSNs and found them significantly correlated $(p<0.05)$. Therefore, to avoid multicollinearity issues, we run the logistic regression including each RSN one by one. The logistic regression models were statistically significant for each RSN investigated ( $p<0.05$ ), explaining a percentage of variance (Nagelkerke $R^{2}$ ) in NEDA status ranging from 36 to $79 \%$, and correctly classifying $80-93 \%$ of cases.

\section{Between-Network Connectivity}

No significant differences in partial or full correlations between RSNs were found at Tst between NEDA and EDA patients. Instead, after therapy NEDA patients showed a significantly higher partial correlation between the posterior default mode and orbitofrontal networks ( $p<0.05$, FWE corrected) (Figure 5). Moreover, a positive correlation was found between 

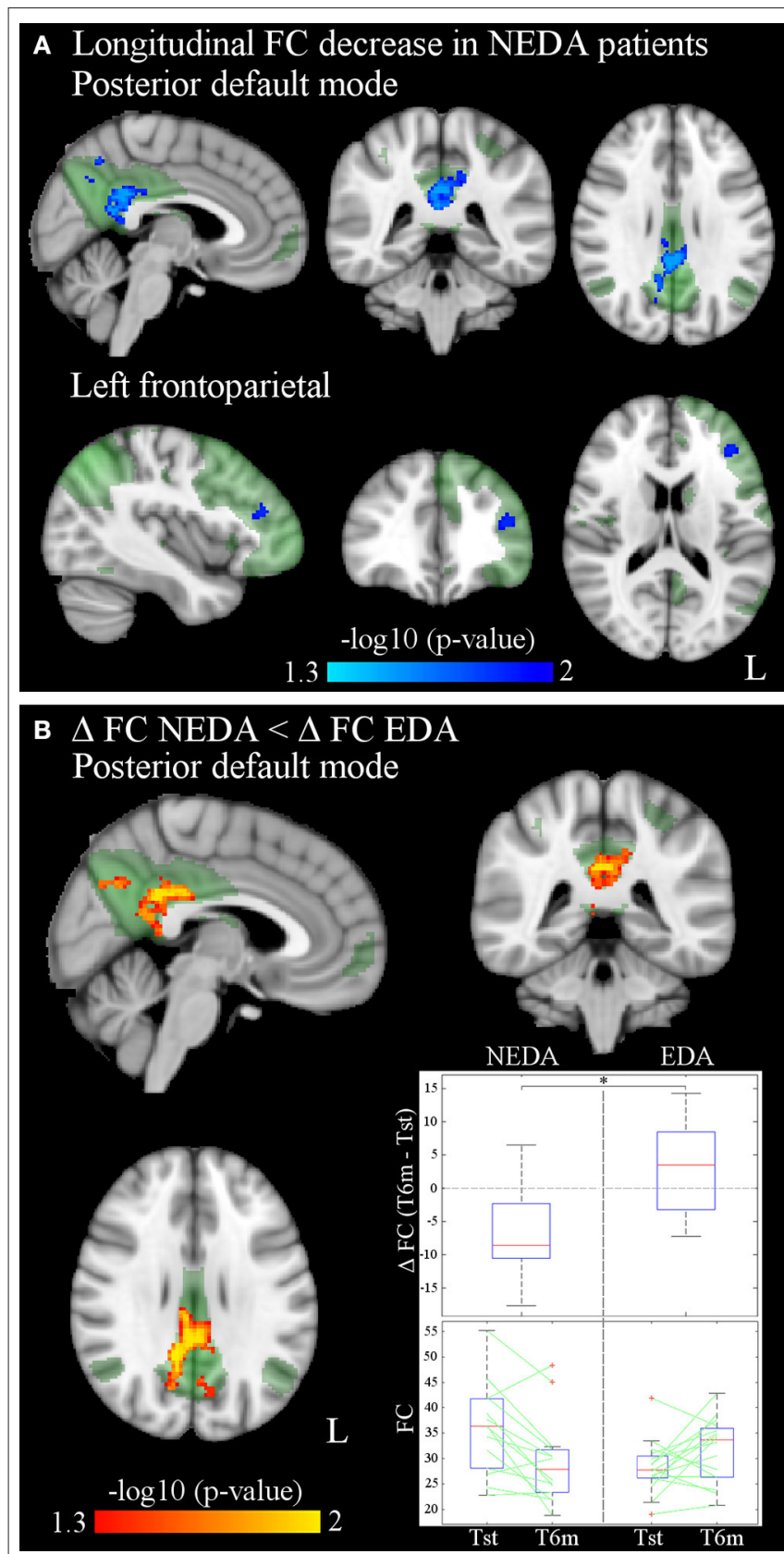

FIGURE 3 | (A) Significantly decreased within-network FC of the posterior default mode and left frontoparietal networks after 6 months of fingolimod in NEDA patients ( $p<0.05$, FWE corrected). Results for each RSN are overlaid onto the corresponding network (green) in the MNI152 standard brain. The blue/light-blue color bar represents level of significance. The -log10(p)-value represents the negative base-10 logarithm of the $p$-value. A larger value of $-\log 10(p)$ corresponds to a smaller $p$-value. (B) Significant differences between $\triangle F C$ in NEDA and EDA patients ( $p<0.05$, FWE corrected): $\triangle F C$ in NEDA is significantly lower than $\triangle F C$ in EDA patients. The red-yellow color bar represents level of significance. The box plots show the distribution of $\triangle F C$ (T6m-Tst) values of NEDA and EDA patients, and the individual values at Tst and T6m for each group inside the mask of between-group differences. On each box, the central mark indicates the median, and the bottom and top edges of the box indicate the 25th and 75th percentiles, respectively. The whiskers extend to the most extreme data points not considered outliers, and the outliers are plotted individually using the "+" symbol.
TABLE 3 | RSNs showing significant within-network FC reduction after 6 months of fingolimod therapy in NEDA patients ( $p<0.05$, FWE corrected).

\section{MNI coordinates}

Cluster size (voxels) $\quad p \quad x \quad y \quad z \quad$ Cluster location (local maxima)

Posterior default mode network

656

$$
\begin{array}{lclll}
0.018 & 2 & -42 & 28 & \text { Cingulate gyrus, posterior division } \\
0.028 & 10 & -60 & 34 & \text { Precuneous cortex } \\
0.042 & -12 & -28 & 36 & \text { Cingulate gyrus, posterior division }
\end{array}
$$

Left frontoparietal network

52

$0.038-40 \quad 38 \quad 18$ Left frontal pole/middle frontal gyrus

Refer to Table 2 for a detailed explanation of the table layout

TABLE 4 | Significant within-network FC differences between $\triangle F C$ maps of NEDA and EDA patients in the posterior default mode network $(p<0.05$, FWE corrected).

\section{MNI coordinates}

$\begin{array}{lllllll}\text { Cluster size (voxels) } & p & x & y & z & \text { Cluster location (local maxima) }\end{array}$

\section{Posterior default mode network}

$987 \quad 0.011 \quad 10 \quad-48 \quad 26 \quad$ Cingulate gyrus, posterior division

$0.020-10-54 \quad 34$ Precuneous cortex

$0.025 \quad 6 \quad-72 \quad 32$ Precuneous cortex

$\triangle F C$ in NEDA patients was significantly lower than $\triangle F C$ in EDA patients. Refer to Table 2 for a detailed explanation of the table layout.

$\triangle$ PASAT3 and FC changes in the posterior default mode network/orbitofrontal network correlation (Spearman rho $=$ $0.61, p=0.048)$; the greater the PASAT improvement, the greater the FC increase (Figure 5). No significant differences in partial or full correlations between RSNs were found in EDA patients after therapy.

\section{DISCUSSION}

In the present postmarketing study, we analyzed data from a previously published dataset on relapsing MS patients who underwent fingolimod treatment for 6 months (9), using the NEDA-3 status characterization as a clinical response indicator of treatment efficacy.

We found significant baseline differences in within-network FC between NEDA and EDA patients in several RSNs, with NEDA patients showing higher FC than EDA patients. Furthermore, only NEDA patients showed significant changes in within- and between-network FC after fingolimod. These longitudinal changes mainly regarded the posterior default mode network and correlated with a significant improvement in PASAT score.

\section{Epidemiological Coherence}

In our study, almost half of patients achieved NEDA after 6 months of treatment $(46.6 \%)$. This result is consistent with realworld studies on large populations of relapsing MS patients 

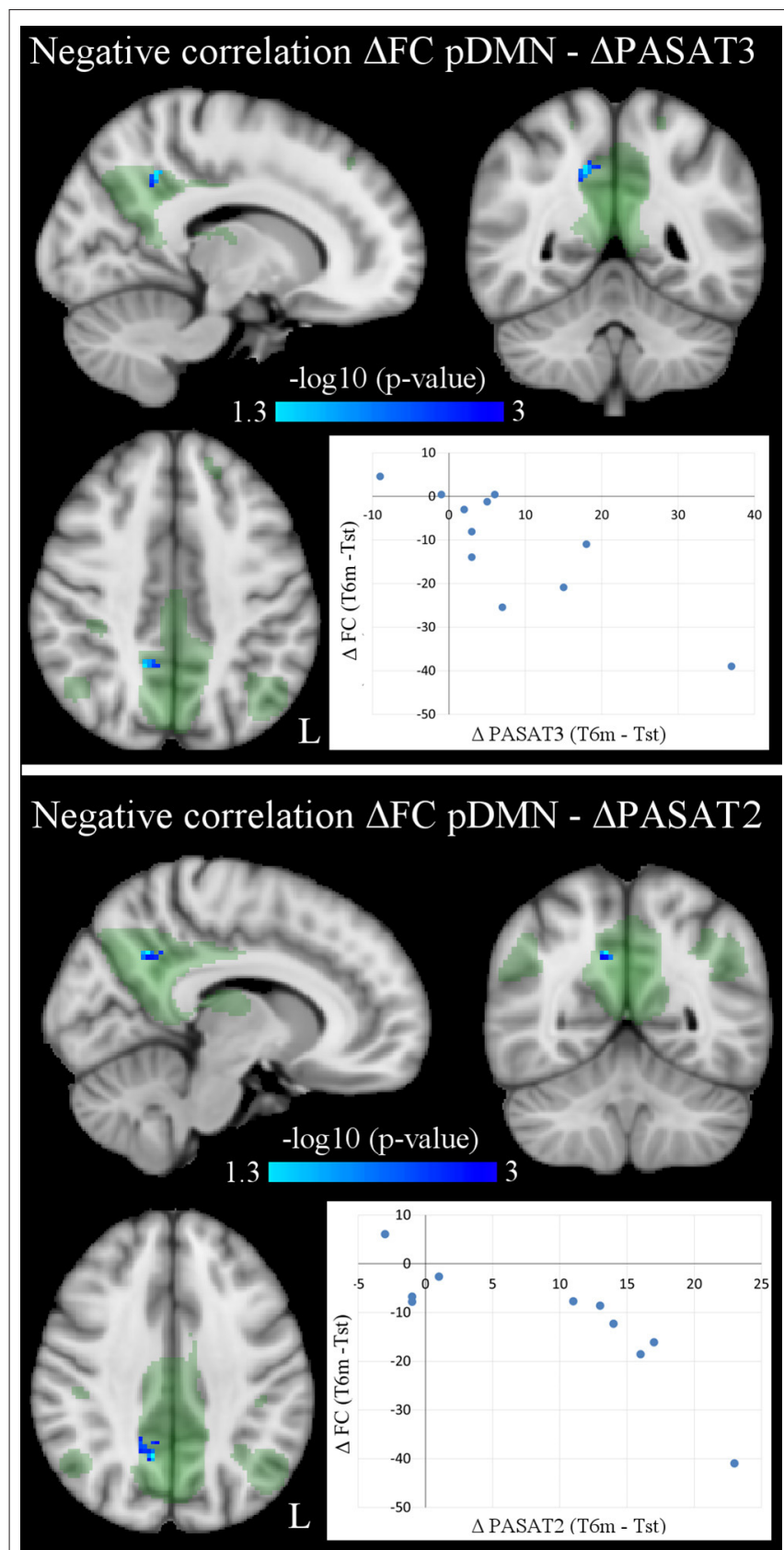

FIGURE 4 | Voxel-wise negative correlations in NEDA patients between within-network FC changes in posterior default mode network (pDMN) and PASAT improvement ( $\triangle$ PASAT3 and $\triangle$ PASAT2) after 6 months of fingolimod ( $p<0.05$, FDR corrected). As shown in the scatterplots, the greater the PASAT increase, the greater the FC decrease ( $\triangle F C$ values obtained at cluster local maxima). Results are overlaid onto the posterior default mode network map (green) in the MNI152 standard brain. The blue/light-blue color bar represents level of significance. The -log10(p)-value represents the negative base-10 logarithm of the $p$-value. A larger value of $-\log 10(p)$ corresponds to a smaller $p$-value.

receiving fingolimod that have reported that $35-60 \%$ of patients achieve NEDA-3 status over 1-2 years of treatment $(31,32)$. We did not find any significant differences in radiological or
TABLE 5 | Significant negative correlations between longitudinal FC changes in the posterior default mode network $(\triangle \mathrm{pDMN})$ and PASAT improvements ( $\triangle$ PASAT3 and $\triangle$ PASAT2) in NEDA patients $(p<0.05$, FDR corrected).

\begin{tabular}{|c|c|c|c|c|c|}
\hline \multirow[b]{2}{*}{ Cluster size (voxels) } & \multirow[b]{2}{*}{$p$} & \multicolumn{3}{|c|}{ MNI coordinates } & \multirow[b]{2}{*}{ Cluster location (local maxima) } \\
\hline & & $x$ & $y$ & $z$ & \\
\hline \multicolumn{6}{|l|}{$\Delta$ pDMN- $\triangle$ PASAT3 $\downarrow$} \\
\hline 22 & 0.001 & 14 & -50 & 42 & Precuneous cortex \\
\hline \multicolumn{6}{|l|}{$\Delta$ pDMN- $\triangle$ PASAT2 $\downarrow$} \\
\hline 46 & $<0.001$ & 10 & -56 & 36 & Precuneous cortex \\
\hline
\end{tabular}

Refer to Table 2 for a detailed explanation of the table layout.

neuropsychological variables between NEDA and EDA patients at baseline. However, we found a significantly higher number of males in the NEDA group. Regarding treatment-by-sex interaction, literature shows inconsistent findings [see (33) for details] and the small number of males in the present study did not allow further generalization.

\section{Predictive Value of Baseline Functional Connectivity for Treatment Response}

The search for predictive biomarkers in MS treatment is ongoing. In fingolimod treatment, some recent studies have found different baseline variables associated with a higher likelihood of achieving NEDA. Giuliani and colleagues (34) reported that baseline clinical factors, such as a higher baseline EDSS score and more relapses in the year before the start of fingolimod were highly associated with the risk of not achieving NEDA. Other authors have reported that baseline parameters of higher disease activity (annualized relapse rate, gadolinium-enhancing lesions, and age at onset) were associated with an increased likelihood of failing to achieve NEDA criteria (32).

This is the first study that investigates and reports significant baseline FC differences between patients who achieved NEDA and those who did not. Of note, no other radiological parameter at baseline differed between NEDA and EDA patients. Accordingly, with reference to the possible effect of an established DMT therapy, we can hypothesize a specific predictive value of baseline within-network FC for NEDA after a time lapse of 6 months.

\section{Functional Reserve Engagement as a Putative Mechanism for NEDA Prediction}

We hypothesize that functional reserve could play a role in baseline differences. According to Medaglia et al. (35), "reserve represents individual variability in the functional use or structural integrity of the nervous system that alters a person's cognitive and behavioral abilities following the onset of brain pathology. In essence, the concept of reserve suggests that some initial conditions of brain physiology and function - often measured using neuroimaging techniques-heavily constrain the observed clinical sequelae." Consistent with an increasing number of studies suggesting that RSN organization is associated with reserve level and reflects the influence of reserve on brain network organization (36), in our study, we found that a higher 

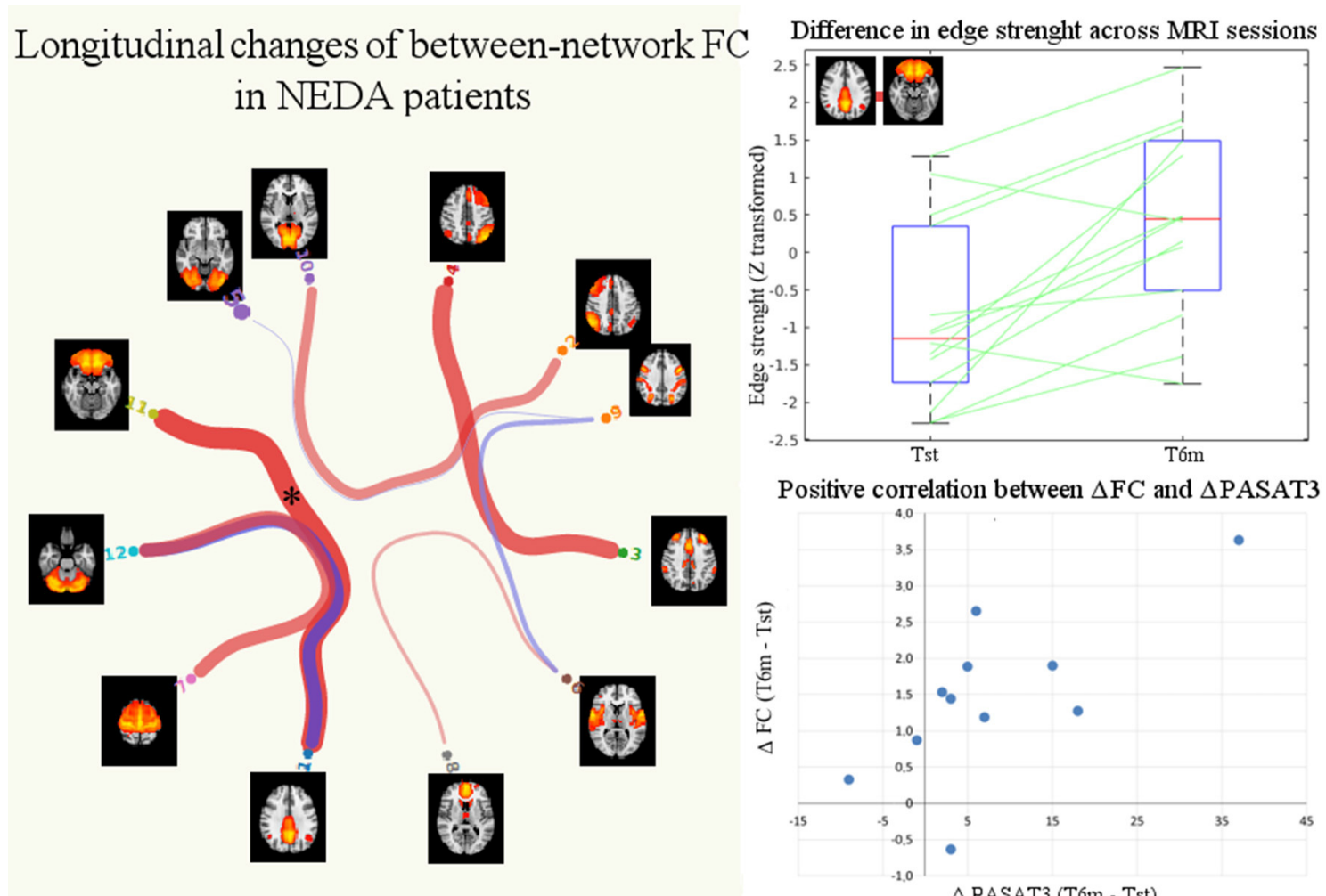

Positive correlation between $\triangle \mathrm{FC}$ and $\triangle \mathrm{PASAT} 3$

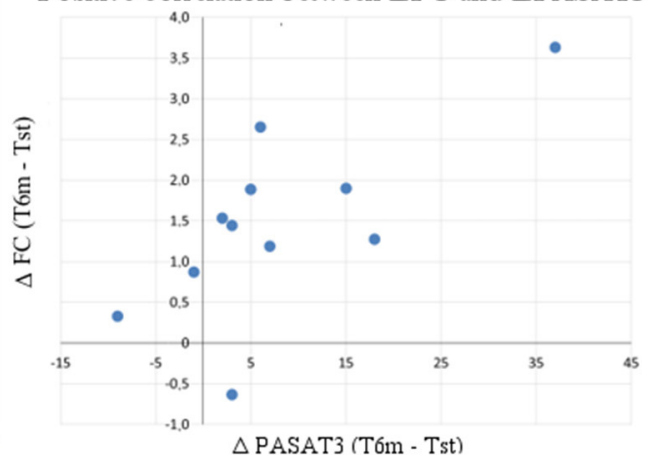

FIGURE 5 | Significant changes in between-network FC in NEDA patients after 6 months of fingolimod. The NEDA subgroup showed a significantly higher correlation between the posterior default mode and orbitofrontal networks ( $p<0.05$ FWE corrected, indicated with asterisk). The box plot shows the distributions of the correlation values (edge strengths) at Tst and T6m in NEDA patients. On each box, the central mark indicates the median, and the bottom and top edges of the box indicate the 25th and 75th percentiles, respectively. The whiskers extend to the most extreme data points. The scatterplot shows the positive correlation between posterior default mode/orbitofrontal networks increased FC ( $\triangle F C$, expressed as the difference between T6m and Tst of the z-transformed correlation coefficients) and PASAT improvement ( $\triangle$ PASAT3).

connectivity at baseline could characterize MS patients with a higher functional reserve. Indeed, previous studies have linked altered FC with reduced or preserved functional reserve in MS (37-39).

\section{Fingolimod Treatment Reduces Baseline High Functional Connectivity}

At the longitudinal level, we found significant FC changes in NEDA patients, suggesting that pharmacological modulation promotes a more favorable reorganization only in those with a more efficient functional reserve. However, it is not fully understood how immunomodulation modifies recovery and connectivity. The anti-inflammatory and immunomodulatory effects of fingolimod may result in the restoration of synaptic plasticity and, in turn, the facilitation of recovery $(40,41)$.

Longitudinal connectivity changes after 6 months of fingolimod treatment were found in cognitive-related RSNs (posterior default mode, left frontoparietal, and orbitofrontal) and were especially evident in specific regions of the posterior default mode network, namely the precuneus and posterior cingulate cortex. These regions are involved in cognitive function and act as network hubs whose connectivity is modulated in relation to several brain networks (42). The significant changes in these regions are also consistent with findings from our previous work, in which we reported a longitudinal fingolimod-related FC decrease between these posterior cortical areas and the cortical area of the right hand (9).

\section{Evidence of Favorable Cognitive Function Modulation After Fingolimod}

Connectivity changes in the posterior default mode network after fingolimod treatment correlated with a PASAT score increase, reflective of an improvement in information processing speed associated with the effects of the drug. In MS, modulation of the default mode network has previously been found to be related to improvement in cognitive tasks after training $(43,44)$.

Despite the ongoing debate about the adaptive or maladaptive role of functional connectivity changes in the context of MS (45), our finding of decreased FC after fingolimod can be interpreted as a positive phenomenon, in line with previous studies that reported increased functional connectivity in the default mode network associated with poorer performance in cognitive tests (46-48). 
One concern is that the observed improvement in PASAT scores and concomitant FC changes of NEDA patients are, at least in part, due to a practice or learning effect. A recent study suggested that short-term practice effects on PASAT are related to brain volume, disease severity, and age and have clinically meaningful prognostic implications. High learners benefited more from fingolimod treatment (49). Therefore, we suggest that an increase in PASAT score, implying cognitive improvement and/or the retention of practice effects, can be interpreted as a positive phenomenon. It should also be noted that PASAT is particularly sensitive to fingolimod treatment (50) and is a difficult and demanding test that is even sensitive to early cognitive changes in patients where room for improvement is still limited $(51,52)$.

\section{Study Limitations}

One of the main limitations of the present study is the small sample size, along with the lack of a control group of healthy subjects or a group of untreated patients. Although comparison with healthy subjects would have allowed us to characterize the observed FC changes as patient specific, previous studies have shown that MS patients and healthy subjects have different baseline connectivity in most RSNs (53). The longitudinal study design was also meant to compensate for the lack of an untreated MS patient group, which would be unethical. As reported above, baseline clinical and MRI features did not differ between the 2 subgroups in the present study. However, due to the small study sample, we cannot exclude that mechanisms of compensation could take place mainly in patients with less baseline disease activity and less clinical disease burden, as previous studies seem to suggest $(32,34)$. For the same reason, although we did not find significant subgroup differences in the number of naïve or switching patients (either first- or second-line DMTs), we cannot exclude the relevance of this condition on FC modulation.

Another limitation of the present study is that the antiinflammatory effects of fingolimod were evaluated only by means of conventional MRI. The use of other techniques, such as positron emission tomography, would have assessed the inflammation of brain tissue outside the lesion areas (54).

A further limitation is the lack of physiological data recordings (e.g., through pulse-oximeter, respiratory bellows, expired gas analyzer) as well as more direct measures of perfusion (e.g., ASL) or vascular reactivity (e.g., breath-holding task). Although the existence of different data-driven methods, physiological noise correction in fMRI data has not emerged as a standard preprocessing step (55), and in the present clinical study, we opted for a more standard pipeline.

The longer-term effects of fingolimod have not been evaluated, and a re-baselining was not performed. Additionally, NEDA outcome is usually evaluated after 1-2 years, and this timeframe would also have allowed us to measure the annualized percentage of brain volume loss and progression to NEDA-4 status. Nevertheless, our percentage of patients with NEDA-3 status at 6 months is consistent with literature. Furthermore, a 6month observation time was also selected to avoid as much as possible the confounding effects of neurodegeneration (atrophy modifications can take years to occur and brain volume measures acquired in the first period of treatment can be affected by several factors, i.e., pseudoatrophy [see (4)]. Finally, cognitive status was assessed with only the PASAT score, which cannot be considered a global measure of cognitive function. However, the PASAT score has proven its validity in detecting even early cognitive impairment and sensitivity to treatment-related modifications [see (9)].

\section{CONCLUSION}

The present study showed that in MS, the achievement of NEDA status after disease-modifying treatment may be predicted by higher FC at baseline and is associated with significant brain functional rearrangement after 6 months of therapy. These functional changes occurred in cognitive-related networks and were paralleled by an improvement in information processing speed. FC changes in this initial treatment period may reflect neuroplastic changes promoted by a more efficient functional reserve through the effects of fingolimod. Although the use of $\mathrm{fMRI}$ as a tool in clinical decision-making in individual patients seems a long way off, our study suggests that a similar estimate of functional reserve is promising potential predictor of clinical outcome.

\section{DATA AVAILABILITY STATEMENT}

The datasets presented in this article are not readily available because of patient confidentiality and participant privacy restrictions. Requests to access the datasets should be directed to petsas@gmail.com.

\section{ETHICS STATEMENT}

This study, involving human participants, was reviewed and approved by the ethics committee of Azienda Policlinico Umberto I, Sapienza University of Rome. The patients/participants provided their written informed consent to participate in this study.

\section{AUTHOR CONTRIBUTIONS}

CPi contributed to method definition, data analysis, statistics, and manuscript editing. NP contributed to study design, experimental settings, subject recruitment, MRI acquisition, and manuscript editing. LD, ST, and CG contributed to data interpretation and manuscript editing. MC performed MRI acquisition. CPo contributed to study design and recruitment and, together with PP, supervised the study and manuscript editing. All authors contributed to the article and approved the submitted version.

\section{SUPPLEMENTARY MATERIAL}

The Supplementary Material for this article can be found online at: https://www.frontiersin.org/articles/10.3389/fneur. 2021.632917/full\#supplementary-material 


\section{REFERENCES}

1. Reich DS, Lucchinetti CF, Calabresi PA. Multiple sclerosis. $N$ Engl J Med. (2018) 378:169-80. doi: 10.1056/NEJMra1401483

2. Giovannoni G. Disease-modifying treatments for early and advanced multiple sclerosis: a new treatment paradigm. Curr Opin Neurol. (2018) 31:23343. doi: 10.1097/WCO.0000000000000561

3. Comi G, Radaelli M, Soelberg Sørensen P. Evolving concepts in the treatment of relapsing multiple sclerosis. Lancet. (2017) 389:1347-56. doi: 10.1016/S0140-6736(16)32388-1

4. Rocca MA, Preziosa P, Filippi M. Application of advanced MRI techniques to monitor pharmacologic and rehabilitative treatment in multiple sclerosis: current status and future perspectives. Expert Rev Neurother. (2019) 19:83566. doi: 10.1080/14737175.2019.1555038

5. Rocca MA, Agosta F, Colombo B, Mezzapesa DM, Falini A, Comi G, et al. fMRI changes in relapsing-remitting multiple sclerosis patients complaining of fatigue after IFN $\beta$-1a injection. Hum Brain Mapp. (2007) 28:37382. doi: 10.1002/hbm.20279

6. Tomassini V, d'Ambrosio A, Petsas N, Wise RG, Sbardella E, Allen M, et al. The effect of inflammation and its reduction on brain plasticity in multiple sclerosis: MRI evidence: inflammation and brain plasticity in MS. Hum Brain Mapp. (2016) 37:2431-45. doi: 10.1002/hbm.23184

7. Fox MD, Raichle ME. Spontaneous fluctuations in brain activity observed with functional magnetic resonance imaging. Nat Rev Neurosci. (2007) 8:70011. doi: $10.1038 / \mathrm{nrn} 2201$

8. Greicius M. Resting-state functional connectivity in neuropsychiatric disorders. Curr Opin Neurol. (2008) 21:42430. doi: 10.1097/WCO.0b013e328306f2c5

9. Petsas N, De Giglio L, González-Quintanilla V, Giuliani M, De Angelis F, Tona F, et al. Functional connectivity changes after initial treatment with fingolimod in multiple sclerosis. Front Neurol. (2019) 10:153. doi: 10.3389/fneur.2019.00153

10. Giovannoni G, Turner B, Gnanapavan S, Offiah C, Schmierer K, Marta M. Is it time to target no evident disease activity (NEDA) in multiple sclerosis? Mult Scler Relat Disord. (2015) 4:329-33. doi: 10.1016/j.msard.2015.04.006

11. Polman CH, Reingold SC, Banwell B, Clanet M, Cohen JA, Filippi M, et al. Diagnostic criteria for multiple sclerosis: 2010 revisions to the MCDONALD criteria. Ann Neurol. (2011) 69:292-302. doi: 10.1002/ana.22366

12. Kurtzke JF. Rating neurologic impairment in multiple sclerosis: an expanded disability status scale (EDSS). Neurology. (1983) 33:1444-52.

13. Fischer JS, Rudick RA, Cutter GR, Reingold SC. The multiple sclerosis functional composite measure (MSFC): an integrated approach to MS clinical outcome assessment. National MS society clinical outcomes assessment task force. Mult Scler. (1999) 5:244-50. doi: 10.1177/135245859900500409

14. Esteban O, Birman D, Schaer M, Koyejo OO, Poldrack RA, Gorgolewski KJ. MRIQC: advancing the automatic prediction of image quality in MRI from unseen sites. PLOS ONE. (2017) 12:e0184661. doi: 10.1371/journal.pone.0184661

15. Jenkinson M, Bannister P, Brady M, Smith S. Improved optimization for the robust and accurate linear registration and motion correction of brain images. Neuroimage. (2002) 17:825-41. doi: 10.1016/s1053-8119(02)91132-8

16. Smith SM. Fast robust automated brain extraction. Hum Brain Mapp. (2002) 17:143-55. doi: 10.1002/hbm.10062

17. Jenkinson M, Smith S. A global optimisation method for robust affine registration of brain images. Med Image Anal. (2001) 5:143-56. doi: 10.1016/S1361-8415(01)00036-6

18. Greve DN, Fischl B. Accurate and robust brain image alignment using boundary-based registration. Neuroimage. (2009) 48:6372. doi: 10.1016/j.neuroimage.2009.06.060

19. Andersson JL, Jenkinson M, Smith S. Non-linear registration, aka spatial normalisation FMRIB technical report TR07JA2. (2007) Available online at: www.fmrib.ox.ac.uk/analysis/techrep (accessed February 10, 2021).

20. Beckmann CF, DeLuca M, Devlin JT, Smith SM. Investigations into restingstate connectivity using independent component analysis. Philos Trans R Soc Lond B Biol Sci. (2005) 360:1001-13. doi: 10.1098/rstb.2005.1634

21. Smith SM, Fox PT, Miller KL, Glahn DC, Fox PM, Mackay $\mathrm{CE}$, et al. Correspondence of the brain's functional architecture during activation and rest. Proc Natl Acad Sci USA. (2009) 106:13040-5. doi: 10.1073/pnas.0905267106
22. Tong Y, Hocke LM, Nickerson LD, Licata SC, Lindsey KP, Frederick B deB. Evaluating the effects of systemic low frequency oscillations measured in the periphery on the independent component analysis results of resting state networks. Neuroimage. (2013) 76:202-15. doi: 10.1016/j.neuroimage.2013.03.019

23. Yeo BTT, Krienen FM, Sepulcre J, Sabuncu MR, Lashkari D, Hollinshead $\mathrm{M}$, et al. The organization of the human cerebral cortex estimated by intrinsic functional connectivity. J Neurophysiol. (2011) 106:112565. doi: 10.1152/jn.00338.2011

24. Filippini N, MacIntosh BJ, Hough MG, Goodwin GM, Frisoni GB, Smith SM, et al. Distinct patterns of brain activity in young carriers of the APOE-epsilon4 allele. Proc Natl Acad Sci USA. (2009) 106:720914. doi: 10.1073/pnas.0811879106

25. Nickerson LD, Smith SM, Öngür D, Beckmann CF. Using dual regression to investigate network shape and amplitude in functional connectivity analyses. Front Neurosci. (2017) 11:115. doi: 10.3389/fnins.2017.00115

26. Power JD, Barnes KA, Snyder AZ, Schlaggar BL, Petersen SE. Spurious but systematic correlations in functional connectivity MRI networks arise from subject motion. Neuroimage. (2012) 59:2142-54. doi: 10.1016/j.neuroimage.2011.10.018

27. Power JD, Schlaggar BL, Petersen SE. Recent progress and outstanding issues in motion correction in resting state fMRI. Neuroimage. (2015) 105:53651. doi: 10.1016/j.neuroimage.2014.10.044

28. Van Dijk KRA, Sabuncu MR, Buckner RL. The influence of head motion on intrinsic functional connectivity MRI. Neuroimage. (2012) 59:4318. doi: 10.1016/j.neuroimage.2011.07.044

29. Nichols TE, Holmes AP. Nonparametric permutation tests for functional neuroimaging: a primer with examples. Hum Brain Mapp. (2002) 15:1-25. doi: $10.1002 /$ hbm. 1058

30. Smith S, Nichols T. Threshold-free cluster enhancement: addressing problems of smoothing, threshold dependence and localisation in cluster inference. NeuroImage. (2009) 44:83-98. doi: 10.1016/j.neuroimage.2008.03.061

31. Weinstock-Guttman B, Medin J, Khan N, Korn JR, Lathi E, Silversteen J, et al. Assessing "no evidence of disease activity" status in patients with relapsing-remitting multiple sclerosis receiving fingolimod in routine clinical practice: a retrospective analysis of the multiple sclerosis clinical and magnetic resonance imaging outcomes in the USA (MS-MRIUS) study. CNS Drugs. (2018) 32:75-84. doi: 10.1007/s40263-017-0482-4

32. Esposito F, Ferrè L, Clarelli F, Rocca MA, Sferruzza G, Storelli L, et al. Effectiveness and baseline factors associated to fingolimod response in a real-world study on multiple sclerosis patients. J Neurol. (2018) 265:896905. doi: 10.1007/s00415-018-8791-1

33. Devonshire V, Havrdova E, Radue EW, O'Connor P, Zhang-Auberson L, Agoropoulou C, et al. Relapse and disability outcomes in patients with multiple sclerosis treated with fingolimod: subgroup analyses of the doubleblind, randomised, placebo-controlled FREEDOMS study. Lancet Neurol. (2012) 11:420-8. doi: 10.1016/S1474-4422(12)70056-X

34. Giuliani M, Logoteta A, Prosperini L, Hirsch MN, Pozzilli C. Baseline characteristics associated with NEDA-3 status in fingolimod-treated patients with relapsing-remitting multiple sclerosis. Mult Scler Demyelinating Disord. (2017) 2:10. doi: 10.1186/s40893-017-0026-2

35. Medaglia JD, Pasqualetti F, Hamilton RH, Thompson-Schill SL, Bassett DS. Brain and cognitive reserve: translation via network control theory. Neurosci Biobehav Rev. (2017) 75:53-64. doi: 10.1016/j.neubiorev.2017. 01.016

36. Fleck JI, Arnold M, Dykstra B, Casario K, Douglas E, Morris O. Distinct functional connectivity patterns are associated with social and cognitive lifestyle factors: pathways to cognitive reserve. Front Aging Neurosci. (2019) 11:310. doi: 10.3389/fnagi.2019.00310

37. Cader S, Cifelli A, Abu-Omar Y, Palace J, Matthews PM. Reduced brain functional reserve and altered functional connectivity in patients with multiple sclerosis. Brain. (2006) 129:527-37. doi: 10.1093/brain/awh670

38. Rocca MA, Absinta M, Ghezzi A, Moiola L, Comi G, Filippi M. Is a preserved functional reserve a mechanism limiting clinical impairment in pediatric MS patients? Hum Brain Mapp. (2009) 30:2844-51. doi: 10.1002/hbm.20712

39. Droby A, Yuen KSL, Muthuraman M, Reitz S-C, Fleischer V, Klein J, et al. Changes in brain functional connectivity patterns are driven by an individual lesion in MS: a resting-state fMRI study. Brain Imag Behav. (2016) 10:111726. doi: $10.1007 / \mathrm{s} 11682-015-9476-3$ 
40. Fukumoto K, Mizoguchi H, Takeuchi H, Horiuchi H, Kawanokuchi J, Jin $\mathrm{S}$, et al. Fingolimod increases brain-derived neurotrophic factor levels and ameliorates amyloid $\beta$-induced memory impairment. Behav Brain Res. (2014) 268:88-93. doi: 10.1016/j.bbr.2014.03.046

41. Miguez A, García-Díaz Barriga G, Brito V, Straccia M, Giralt A, Ginés S, et al. Fingolimod (FTY720) enhances hippocampal synaptic plasticity and memory in Huntington's disease by preventing p75NTR up-regulation and astrocyte-mediated inflammation. Hum Mol Genet. (2015) 24:495870. doi: $10.1093 / \mathrm{hmg} / \mathrm{ddv} 218$

42. Tomasi D, Volkow ND. Association between functional connectivity hubs and brain networks. Cereb Cortex. (2011) 21:2003-13. doi: 10.1093/cercor/bhq268

43. Rocca MA, Valsasina P, Absinta M, Riccitelli G, Rodegher ME, Misci P, et al. Default-mode network dysfunction and cognitive impairment in progressive MS. Neurology. (2010) 74:1252-9. doi: 10.1212/WNL.0b013e3181d9ed91

44. De Giglio L, Tona F, De Luca F, Petsas N, Prosperini L, Bianchi V, et al. Multiple sclerosis: changes in thalamic resting-state functional connectivity induced by a home-based cognitive rehabilitation program. Radiology. (2016) 280:202-11. doi: 10.1148/radiol.2016150710

45. Rocca MA, Filippi M. Functional reorganization is a maladaptive response to injury - YES. Multi Sclerosis J. (2017) 23:191-3. doi: $10.1177 / 1352458516667242$

46. Hawellek DJ, Hipp JF, Lewis CM, Corbetta M, Engel AK. Increased functional connectivity indicates the severity of cognitive impairment in multiple sclerosis. Proc Natl Acad Sci USA. (2011) 108:1906671. doi: $10.1073 /$ pnas. 1110024108

47. Meijer KA, Eijlers AJC, Douw L, Uitdehaag BMJ, Barkhof F, Geurts JJG, et al. Increased connectivity of hub networks and cognitive impairment in multiple sclerosis. Neurology. (2017) 88:2107-14. doi: 10.1212/WNL.0000000000003982

48. Schoonheim MM, Meijer KA, Geurts JJG. Network collapse and cognitive impairment in multiple sclerosis. Front Neurol. (2015) 6:82. doi: 10.3389/fneur.2015.00082

49. Sormani MP, De Stefano N, Giovannoni G, Langdon D, Piani-Meier D, Haering DA, et al. Learning ability correlates with brain atrophy and disability progression in RRMS. J Neurol Neurosurg Psychiatry. (2019) 90:3843. doi: 10.1136/jnnp-2018-319129

50. Comi G, Patti F, Rocca MA, Mattioli FC, Amato MP, Gallo P, et al. Efficacy of fingolimod and interferon beta-1b on cognitive, MRI, and clinical outcomes in relapsing-remitting multiple sclerosis: an 18-month, open-label, raterblinded, randomised, multicentre study (the GOLDEN study). J Neurol. (2017) 264:2436-49. doi: 10.1007/s00415-017-8642-5
51. Forn C, Belenguer A, Belloch V, Sanjuan A, Parcet MA, Avila C. Anatomical and functional differences between the paced auditory serial addition test and the symbol digit modalities test. J Clin Exp Neuropsychol. (2011) 33:4250. doi: 10.1080/13803395.2010.481620

52. Karabudak R, Dahdaleh M, Aljumah M, Alroughani R, Alsharoqi IA, AlTahan AM, et al. Functional clinical outcomes in multiple sclerosis: current status and future prospects. Mult Scler Relat Disord. (2015) 4:192201. doi: 10.1016/j.msard.2015.03.004

53. Rocca MA, Valsasina P, Martinelli V, Misci P, Falini A, Comi G, et al. Largescale neuronal network dysfunction in relapsing-remitting multiple sclerosis. Neurology. (2012) 79:1449-1457. doi: 10.1212/WNL.0b013e31826d5f10

54. Poutiainen P, Jaronen M, Quintana FJ, Brownell A-L. Precision medicine in multiple sclerosis: future of PET imaging of inflammation and reactive astrocytes. Front Mol Neurosci. (2016) 9:85. doi: 10.3389/fnmol.2016. 00085

55. Kasper L, Bollmann S, Diaconescu AO, Hutton C, Heinzle J, Iglesias S, et al. The PhysIO toolbox for modeling physiological noise in fMRI data. J Neurosci Methods. (2017) 276:56-72. doi: 10.1016/j.jneumeth.2016.10.019

Conflict of Interest: The dataset used in the present work comes from a previous study (9) that received financial support from Novartis Farma, Italy. NP received speaker fees from Biogen Idec and mission support from Merck Serono, Genzyme, and Novartis. LD received speaking honoraria from Genzyme and Novartis; travel grants from Biogen Idec, Merck Serono, and Teva; and consulting fees from Genzyme, Merck Serono, and Novartis. CPo received consulting and lecture fees from Sanofi-Aventis, Biogen Idec, Bayer Schering, Merck Serono, and Novartis. $\mathrm{He}$ also received research funding from Novartis, Sanofi-Aventis, Merck Serono, and Bayer Schering. PP received travel funding from Novartis, Genzyme, and Bracco and speaker honoraria from Biogen Idec.

The remaining authors declare that the research was conducted in the absence of any commercial or financial relationships that could be construed as a potential conflict of interest.

Copyright (C) 2021 Piervincenzi, Petsas, De Giglio, Carmellini, Gianni, Tommasin, Pozzilli and Pantano. This is an open-access article distributed under the terms of the Creative Commons Attribution License (CC BY). The use, distribution or reproduction in other forums is permitted, provided the original author(s) and the copyright owner(s) are credited and that the original publication in this journal is cited, in accordance with accepted academic practice. No use, distribution or reproduction is permitted which does not comply with these terms. 